\title{
Demanda de servicios empresariales en la región Junín
}

\section{Demand for business services in the Junín region}

\author{
Marco Antonio Cajas Ríos ${ }^{1}$ \\ ${ }_{1}^{1}$ Universidad Continental
}

\section{RESUMEN}

El objetivo fue determinar la demanda de servicios empresariales del empresariado de la región Junín para la mejora de la competitividad. En la metodología se utilizó el diseño transversaldescriptivo. La muestra fue no probabilística, por cuotas, el tamaño muestral fue de 400 empresas de las provincias de Huancayo, Chupaca, Concepción, Jauja, Tarma, Chanchamayo, Satipo, Junín y Yauli del departamento de Junín en Perú. Se utilizó un cuestionario estructurado para la recolección de información que se aplicó según los componentes necesarios para la investigación. En los resultados se determinó que los empresarios de Junín solicitaron los servicios de desarrollo empresarial en un 38 $\%$; búsqueda de nuevas oportunidades de negocio un $19 \%$; y para promover la capacitación de sus trabajadores en un $14 \%$. En conclusión, las buenas prácticas sobre gestión competitiva generan mejores niveles en los indicadores económicos. Por ello es necesario seguir promoviendo programas de desarrollo empresarial como la capacitación, asistencia técnica, asesorías e información de mercados, permitiendo a las empresas alcanzar sus objetivos hacia un mayor impacto económico y crecimiento de la región.

Palabras clave: Gestión competitiva, empresariado, impacto económico.

\begin{abstract}
The objective was to determine the demand for business services of businessmen from the Junín region to improve competitiveness. In the methodology the cross-descriptive design was used. The sample was non probalistic, by quotas, the sample size was 400 companies from the provinces of Huancayo, Chupaca, Concepcion, Jauja, Tarma, Chanchamayo, Satipo, Junín and Yauli of the department of Junín in Peru. A structured questionnaire was used for the collection of information that was applied according to the components necessary for the investigation. In the results it was determined that the businessmen of Junín requested the business development services in $38 \%$; search for new business opportunities 19 $\%$; and to promote the training of their workers by $14 \%$. In conclusion, good practices on competitive management generate better levels of economic indicators. It is therefore necessary to continue promoting business development programs such as training, technical assistance, counseling and market information, allowing companies to achieve their objectives towards greater economic impact and grown in the region.
\end{abstract}

Keywords: Competitive management, business, economic impact.

Historial del artículo:

Recibido, 02 de febrero de 2016; aceptado, 11 de mayo de 2016; disponible en línea, 15 de julio de 2016.

* Docente de la Universidad Continental

Correo: mcajas@continental.edu.pe 


\section{INTRODUCCIÓN}

Es fundamental que, pari passu al PERX (Plan Estratégico Regional Exportador), se desarrolle en el marco de una Instancia Regional de Competitividad una política orientada a promover la competitividad sistémica regional, incluyendo en este concepto el mejoramiento de la infraestructura, de las competencias laborales, de la gerencia, del fortalecimiento institucional y su articulación entre lo nacional y lo regional, de la internacionalización y promoción de las regiones, del financiamiento y del conocimiento en ciencia y tecnología (Ministerio de Comercio Exterior y Turismo, 2005).

En el ámbito económico, la competitividad suele referirse a empresas, regiones o países como sujetos económicos, de forma que adaptando las anteriores definiciones se podría entender en términos generales que competitividad significa la capacidad de una persona, empresa, región, país, etc. (Medina y otros, 2007).

En los últimos años, el término "competitividad" se ha convertido en el referente obligado del debate económico, hasta el punto de su consideración por numerosos especialistas como el factor clave del éxito. Sin embargo, esta unanimidad no resulta tan evidente cuando se plantea la definición conceptual del vocablo, presentándose múltiples criterios, enfoques y opiniones sobre su significado, determinación del sujeto, métodos y parámetros para su medición o en la explicación de sus factores determinantes (Acosta, 1996).

La competitividad es la tarea del futuro y los ganadores serán aquellos que comprendan el cambio y logren adecuarse oportunamente a la nueva economía del siglo XXI priorizando el desarrollo humano: necesitamos de una nueva estrategia competitiva $y$ de un nuevo liderazgo con visión moderna y global (Indacochea, 2005). La ventaja competitiva no puede ser comprendida viendo a una empresa como un todo. Radica en las muchas actividades discretas que desempeña una empresa en el diseño, producción, mercadotecnia, entrega y apoyo de sus productos. Cada una de estas actividades puede contribuir a la posición de costo, por ejemplo, puede sugerir de fuentes tan disparadas como un sistema de distribución físico de bajo costo, un proceso de ensamble altamente eficiente, o del uso de una fuerza de venta superior (Porter, 1996).

La variabilidad en torno a las empresas que participan en el sector, y especialmente las PyME con diferentes trayectorias de crecimiento, supone que se deba proponer un estudio dedicado en su totalidad a la exploración de los aspectos más significativos de los tipos de relaciones establecidas entre la gestión de la innovación y el desempeño competitivo, aplicado a los actores pertenecientes a la industria manufacturera argentina, teniendo en cuenta que son precisamente aquellos los que cuentan con más posibilidades de iniciar procesos de cambios y reconversión que impacten en su desempeño competitivo (Caresani, 2010).

El contar con una infraestructura vial en adecuadas condiciones coadyuva a la reducción de los costos de transporte, lo que repercute en una mayor competitividad regional. Las perspectivas de crecimiento de Junín son favorables debido a que puede constituir una puerta de salida y entrada para el comercio de bienes de la propia región, de regiones aledañas y de Brasil hacia el Pacífico Oeste (Banco Central de Reserva del Perú, 2013).

La mejora de los procesos y el procedimiento vinculados al inicio, operación y cierre de empresas se ha convertido en una prioridad para el Estado Peruano. Los avances conseguidos en la facilitación de negocios hoy sitúan al Perú por encima de varios otros países de la región (Ministerio de Economía y Finanzas, 2013).

Las ventajas competitivas se crean a partir de la diferenciación del producto y de la reducción de costos; aquí la tecnología, la capacidad de innovación y los factores especializados son vitales (Giorgis, 2009). En los últimos años, el término "competitividad" se ha convertido en el referente obligado del debate económico, hasta el punto de su consideración por numerosos especialistas como el factor clave del éxito (Armendáriz, 2011).

La competitividad del Perú, en el orden micro y macro, ha registrado significativos avances durante el último quinquenio. El desempeño positivo reciente de la competitividad nacional es consistente con el crecimiento económico registrado por el Perú durante la última década (Banco Central de Reserva del Perú, 2013). La práctica del buen gobierno corporativo protege los derechos de los inversionistas y stakeholders. Confianza por parte de los diferentes agentes en las empresas y su actuación, crea un mejor ambiente para realizar negocios, direcciona de manera más eficiente los recursos financieros dirigidos al sector productivo, promueve la competitividad (Bolsa de Valores de Lima, 2014).

Cuando la especialización genera nuevos productos y nuevo conocimiento, ésta deriva en procesos de innovación. En esta brecha el análisis de la competitividad incorpora la variable tecnología, la cual enfatiza la contribución de los conocimientos generados en la competitividad (Santana, 2010). La innovación es uno de los factores de competitividad de mayor impacto en el mercado global actual. El 
sector textiles y confecciones colombiano, pese a ser uno de los de mayor crecimiento y representación en las exportaciones colombianas, debe estar diseñando permanentemente estrategias que apunten al aumento de su competitividad para mantenerse en el escenario internacional (Conde, 2009).

En cuanto a los otros indicadores de actividad económica en la región, destacó el incremento en los despachos de cemento $(4,7 \%)$, aunque a una menor tasa que la observada en febrero pasado; la continuidad en el crecimiento del sector manufacturero (18,3\%), determinado por el reinicio de operaciones de la empresa Doe Run (refinación de minerales) desde agosto 2012, no obstante una menor producción de cemento; del crédito directo total $(7,8 \%)$; crédito de consumo $(12,7 \%)$; y crédito hipotecario (14,5 por ciento). Contrariamente, declinó la inversión pública (-14,7 por ciento) por segundo mes consecutivo; los arribos a los establecimientos de hospedaje (-11,5 por ciento); y el empleo formal en las empresas de más de 10 trabajadores en la ciudad de Huancayo (-1,8 por ciento) (Banco Central de Reserva del Perú, 2014).

El Instituto Peruano de Economía (IPE) presenta por segundo año su Índice de Competitividad Regional INCORE 2014- como parte de su esfuerzo por analizar y dar a conocer la realidad económica y social de las regiones del Perú. Entender la competitividad relativa de las regiones y los factores que la determinan es fundamental para la discusión y decisión de las políticas públicas que deben buscar lograr el progreso regional (Instituto Peruano de Economía, 2014).

El empresariado de la Región Junín promueve el desarrollo de la actividad económica y productiva, esto se ve reflejado en los indicadores económicos de la Región Junín, puesto que el empresariado está gestionando competitivamente sus organizaciones, para su fortalecimiento de sus capacidades internas y hacia un nuevo cambio organizacional, con el objetivo de aprovechar de forma eficiente y eficaz los mercados nacionales e internacionales, de esta manera si se quiere promover un mayor impacto económico es necesario dotar a las empresas con mejores herramientas de gestión para generar mayores niveles de competitividad y crecimiento económico, donde el estado sea un ente facilitador de la inversión privada y del desarrollo de los negocios en la región.

Tabla 1: Empresas encuestadas por provincia y distrito.

\begin{tabular}{lcr}
\hline $\begin{array}{c}\text { Provincias/ } \\
\text { distritos }\end{array}$ & Frecuencia & Porcentaje \\
\hline Huancayo & 280 & $70 \%$ \\
Huancayo & 134 & $33,5 \%$ \\
El Tambo & 130 & $32,5 \%$ \\
Chilca & 16 & $4 \%$ \\
Chupaca & 12 & $3 \%$ \\
Concepción & 12 & $3 \%$ \\
Jauja & 20 & $5 \%$ \\
Tarma & 16 & $4 \%$ \\
Chanchamayo & 24 & $6 \%$ \\
Satipo & 16 & $4 \%$ \\
Yauli & 12 & $3 \%$ \\
Junín & 8 & $2 \%$ \\
\hline Total & 400 & 100 \\
\hline
\end{tabular}

\section{MATERIAL Y MÉTODOS}

Se utilizó el diseño transversal - descriptivo. Como instrumento se aplicó un cuestionario estructurado a las empresas de las provincias de Huancayo (distritos de Huancayo, El Tambo y Chilca), Chupaca,

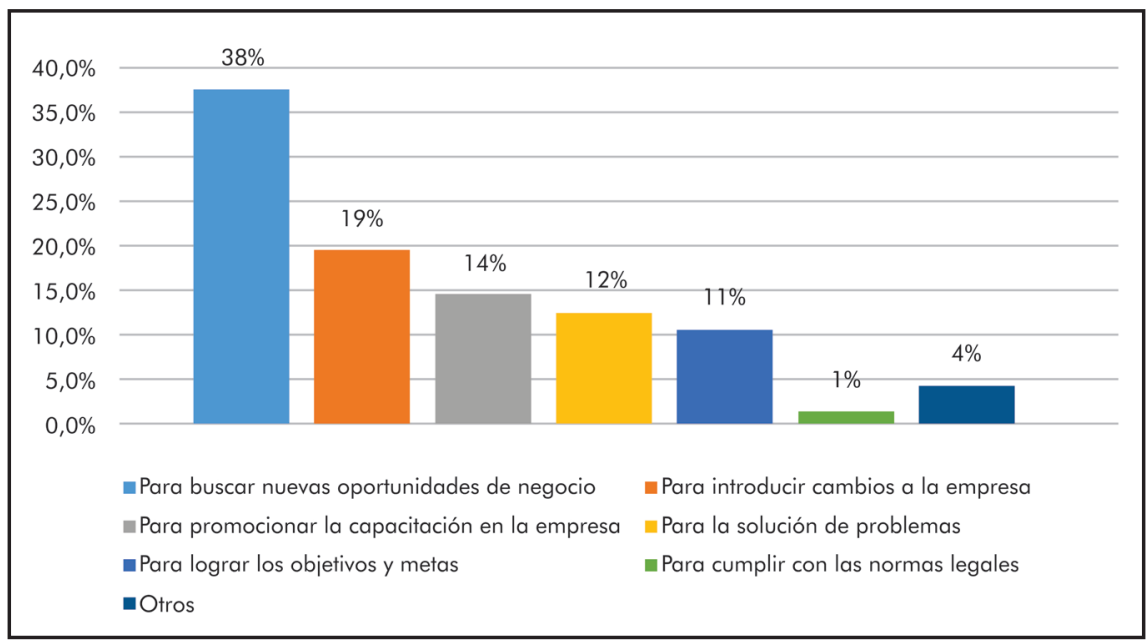

Figura 1: Motivo por el cual las empresas solicitan servicios empresariales en la Región-Junín. 
Concepción, Jauja, Tarma, Chanchamayo, Satipo, Junín y Yauli. Asimismo, se aplicó una guía de entrevista semiestructurada para las entrevistas a las empresas más representativas de los distritos ya mencionados con el fin de observar que tan competitivos están frente al mercado.

\section{RESULTADOS}

Actualmente los empresarios de la Región-Junín utilizan servicios empresariales para buscar nuevas oportunidades de negocio con un $38 \%$; para introducir cambios en la empresa $19 \%$, también para promocionar la capacitación en la empresa con un 14 $\%$, siendo estos tres los motivos más frecuentes por lo que las empresas solicitan los servicios empresariales en la región.

\section{DISCUSIÓN}

Alineado al desarrollo productivo, se ha fortalecido el desarrollo competitivo, asimismo, se han desarrollado instrumentos de apoyo al desarrollo productivo soporte a clusters, programa de proveedores y una plataforma de asistencia técnica y extensión tecnológica, que estarán alineados al Plan de Diversificación Productiva que viene elaborando el Ministerio de la Producción (PRODUCE) con el propósito que las empresas sean más competitivas y sostenibles en el tiempo (Ministerio de Economía y Finanzas, 2013), así también hace mención la investigación realizada, donde los empresarios encuestados mencionan que utilizan servicios empresariales para desarrollar nuevas oportunidades de negocio y para promocionar la capacitación de sus empresas, estos son los factores que generan mayor competitividad a una empresa.

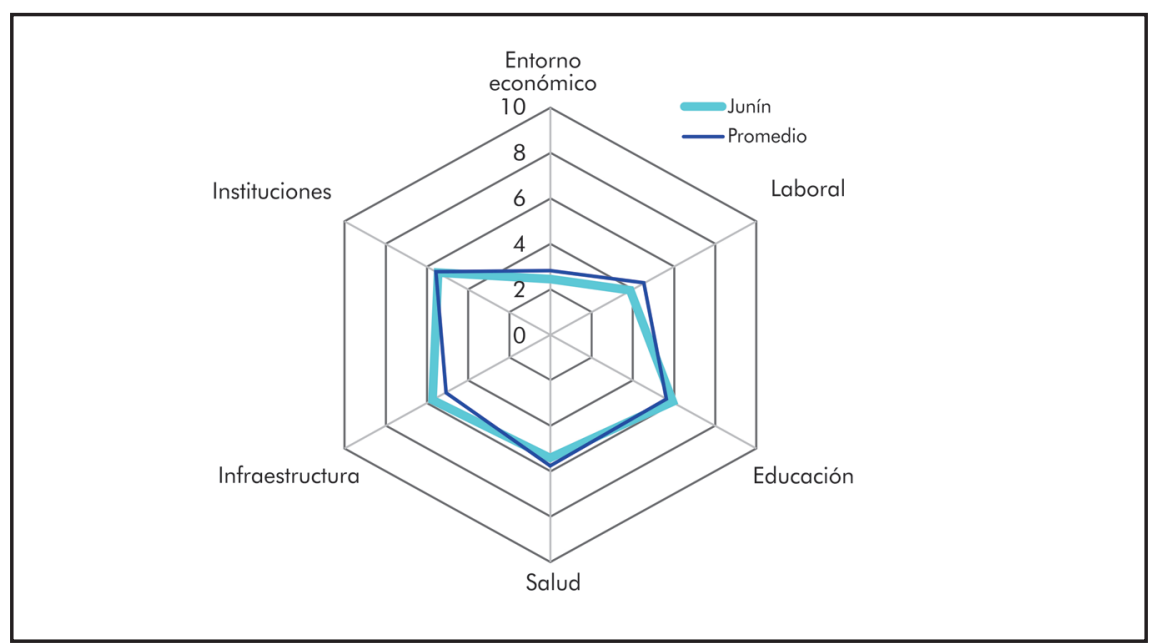

Figura 2: Índice de competitividad regional región-Junín 2014.

Junín ha aumentado un puesto en el índice general. En el pilar Instituciones el porcentaje de ejecución del presupuesto público destinado a inversión ha aumentado. Asimismo la población percibe una mejora en la gestión pública y la seguridad ciudadana. En el pilar Salud ha disminuido el porcentaje de la población que reportó padecer de algún problema de salud.

El INCORE evalúa la competitividad de las 24 regiones del Perú. Su construcción ha considerado un total de 41 indicadores, los cuales han sido agrupados en seis grandes pilares: Entorno Económico, Laboral, Educación, Salud, Infraestructura, Instituciones. La Región-Junín se encuentra en el puesto 14 con respecto al pilar económico y laboral en el promedio del Perú, en educación se ubica en el puesto número 8.
En la nueva economía del conocimiento y la globalización de los mercados la ventaja competitiva ya no radica en la mano de obra barata y los recursos naturales, sino en la capacidad de aprender e innovar, esto es, en el capital intelectual y la competitividad sistemática o integral: empresa-industria-gobiernopaís (Ministerio de Economía y Finanzas, 2009), de esta manera también lo indica el BCRP en su análisis indicadores de competitividad regional Junín, donde se refleja la competitividad de la región, en los indicadores macroeconómicos PBI per cápita, penetración del sistema financiero y del consumo, ya que estos indicadores muestran un avance en las empresas quienes son los principales actores del crecimiento de la región y a competitividad que generan las mismas.

En conclusión la competitividad implica programas de desarrollo productivo, internacionalización de 
las empresas, infraestructura de calidad, eficiencia en procesos administrativos, eliminación de trabas burocráticas, capital humano capacitado y que pueda responder a las necesidades del empresariado, un respeto por nuestra diversidad y medio ambiente, entre otros aspectos. Por tanto, necesitamos una visión sistémica para poder avanzar de manera integral y no esfuerzos aislados del sector público, privado y de la sociedad civil.

\section{REFERENCIAS \\ BIBLIOGRÁFICAS}

Acosta, M. (1996). Factores financieros internos y competitividad empresarial. Barcelona: Universidad de la Laguna.

Armendáriz, E., Jaramillo, F., \& Zegarra, L. (2001). Las barreras al crecimiento económico en Junín $\left(1^{\circ}\right.$ ed.). Junín: Nova Print.

Banco Central de Reserva del Perú. (2013). Competitividad en el Perú. Lima: Banco Central de Reserva del Perú.

Banco Central de Reserva del Perú. (2013). Encuentro económico: Informe económico social. Junín: Banco Central de Reserva del Perú.

Banco Central de Reserva del Perú. (2014). Junín: Síntesis de actividad económica marzo 2014. Junín: Banco Central de Reserva del Perú.

Bolsa de Valores de Lima. (2014). Prácticas de buen gobierno corporativo. Lima: Bolsa de Valores de Lima.
Caresani, D. (2010). Modelos de conductas tecnológicas y su impacto en la competitividad empresarial: El caso de las PYME industriales argentinas. Valencia: Universidad Politécnica de Valencia.

Conde, J. (2009). La innovación como determinación de competitividad en la industria textil. Bogotá: Pontificia Universidad Javeriana.

Giorgis, A. (2009). Factores que afectan la competitividad de las empresas agropecuarias de la zona norte de la provincia de la pampa. Córdoba: Universidad de Córdoba.

Indacochea, A., Ascencio, O., Carranza, F., de los Rios, L., \& Wendorff, J. (2005). Junín competitivo: Valle del Mantaro ( $1^{\circ}$ ed.). Junín: Páginas del Perú.

Instituto Peruano de Economía. (2014). Índice de competitividad regional 2014. Lima: Instituto Peruano de Economía.

Medina, U., \& Gonzáles , A. (2007). Aproximación a los factores determinantes de la competitividad de la empresa de distribución comercial. Barcelona: Universidad de la Laguna.

Ministerio de Comercio Exterior y Turismo. (2005). Plan estratégico regional exportador: Proyecto crecer. Lima: Ministerio de Comercio Exterior y Turismo.

Ministerio de Economía y Finanzas. (2013). 7 pasos a la competitividad. Lima: Ministerio de Economía y Finanzas.

Porter, M. (1996). Ventaja competitiva (3 ed.). Ciudad de México: Continental.

Santana, T. (2010). Innovación y competitividad en la industria azucarera de México. Ciudad de México: Instituto Politécnico Nacional. 